\title{
3D Simulations of the Solar Corona using Octree Compression
}

\author{
F. Saez, P. Lamy and A. Llebaria \\ Laboratoire d'Astrophysique de Marseille, Traverse du Sihpon - BP 8, \\ 13376 Marseille Cedex 12
}

\begin{abstract}
We present a new powerful tool to simulate the streamer belt of the solar corona based on forward modeling. It takes into account the temporal evolution of the corona and provides both qualitative and quantitative results. Starting from the National Solar Observatory photospheric magnetograms, the position of the neutral line at the source surface (2.5 Rsun) is caculated using the potential field source surface model. The plasma sheet of the streamer belt is centered around the current sheet represented as the radial extension of the neutral line. The 3D electron density is represented with octree compression and the radiance images are computed by a ray-tracing algorithm implementing the Thomson scattering. A multi-octree method allows to simulate the temporal evolution of the streamer belt and to compute the synoptic maps from time-series of generated images. The comparison between the synoptic maps of the streamer belt obtained with the SOHO/LASCO-C2 coronagraph and the simulated synoptic maps constructed from our model shows a global agreement for both radiance profiles and global behaviour of the streamer and confirms earlier findings by Wang et al. (1997) that the streamers are associated with folds in the plasma sheet. However, some features cannot be explained using this method and are interpreted by introducing two types of large-scale structures. Our results suggest that the potential field source surface model is not fully adequate for the description of the fine structure of the streamer belt, even during the time of low solar activity. We present new applications of our method to future coronographic observations with SECCHI/COR-2 on STEREO and SILC on Solar Orbiter.
\end{abstract}

Keywords. Sun: activity, Sun: corona, Sun: evolution, Sun: magnetic fields

\section{Introduction}

The solar corona is dominated by the coronal holes and the streamer belt. The simulation of the latter still remains problematic because of its time evolution and the solar activity. Past simulations are either purely qualitative (Koutchmy et al. 1996, Wang et al. 1997 and Wang et al. 2000), or quantitative (Koutchmy 1971, Saito et al. 1972). We propose a new forward modeling method to simulate the streamer belt, which allows both qualitative and quantitative results, in the different activity phasis and takes into account the time evolution of the solar corona. This method, developped for the anaysis of LASCO-C2 images, can also be used for the simulation of the corona in the scope of future coronagraphs : SECCHI/COR-2 (STEREO) and SILC (Solar Orbiter).

Our method relies on the concept of a streamer belt associated to the neutral magnetic surface, so called neutral sheet (Schatten et al. 1969). The continuous sheet of plasma is symmetrically distributed in a thin layer around the neutral sheet. The streamer belt is assumed to be in solid body in rotation with the Sun. Its structures are linked to the warping of the neutral sheet via projection effects (Bohlin and Garrison 1974). 


\section{The method}

The computation of the neutral sheet is performed using the Potential Field Source Surface model (PFSS) introduced by Schatten et al. (1969). The neutral sheet is determined from the magnetic field at the source surface $\left(2.5 R_{\text {sun }}\right)$ which is derived from $\mathrm{NSO} \dagger$ photospheric magnetic field maps using the Current free approximation. Outward the source surface, the magnetic field is purely radial.

The 3D electron density is described by the product of two independant functions. The first one describes its radial variation $N_{1}(r)$ where $r$ is the radial distance to the Sun The second one describes its thickness as a function $N_{2}(d)$ where $d$ is the distance to the neutral sheet. The $3 \mathrm{D}$ resulting electron density is compressed using the octree compression. It is an adaptative resolution allowing the subdivisions proportional to the gradient of the electron density. The computed images are performed using a ray tracing algorithm through the octree, implementing the Thomson scattering. To simulate a full Carrington rotation (CR) taking into account the time evolution, we use a multi octree method. Nine octrees are generated corresponding to 9 different dates regularly sampling a CR, meaning that 1 octree covers an interval of 3 days. The images are computed by the rotations of the projection plane over this interval.

\section{Results}

The present method was mainly elaborated in the scope of the SOHO mission to interprete LASCO-C2 images. But it can easily be applied to other missions such as STEREO and Solar Orbiter.

\subsection{The SOHO mission : LASCO-C2}

The qualitative results are shown in figure 1. It displays two examples of synoptic maps corresponding to the minimum and the maximum of activity. They are computed from observations at $2.5 R_{\text {sun }}$ and at $3.5 R_{\text {sun }}$ (top), from calculated images at $2.5 R_{\text {sun }}$ (middle). The mean configuration of the source surface magnetic field computed from PFSS model are displayed in the bottom. It shows that the global behaviour of the streamer belt is correctly simulated for the CR 1911. The simulation of the CR 1955 reveals some additional structures which are not simulated contrary to the coronal holes which are correctly located. To simulate the additionnal structures, two method are proposed (Saez et al. 2005): the fisrt one is an additional fold of the neutral sheet, and the second one is a plasma sheet with ramification in the form of a secondary short plasma sheet.

Figure 2 displays some radiance profiles extracted from the synoptic maps. The plots shows that the radiance levels are in good agreement for both minimum and maximum of activity. However the right plots particularly reveals two main discrepancies: a small shift of the streamer and some additional structures which are not simulated.

\subsection{The STEREO Mission: SECCHI/COR-2}

Figure 3 shows the evolution of the simulated images of the two STEREO COR-2 coronagraphs as function of time and angular separation. Note the slightly different configuration of the solar corona as the angular separation increases.

\subsection{The Solar Orbiter mission: SILC}

Our method can be applied to simulate the streamer belt seen at any heliocentric distance and at any heliographic latitude. Figure 4 illustrates the cases where the Solar Orbiter

$\dagger$ National Solar Observatory http://www.nso.edu/ 

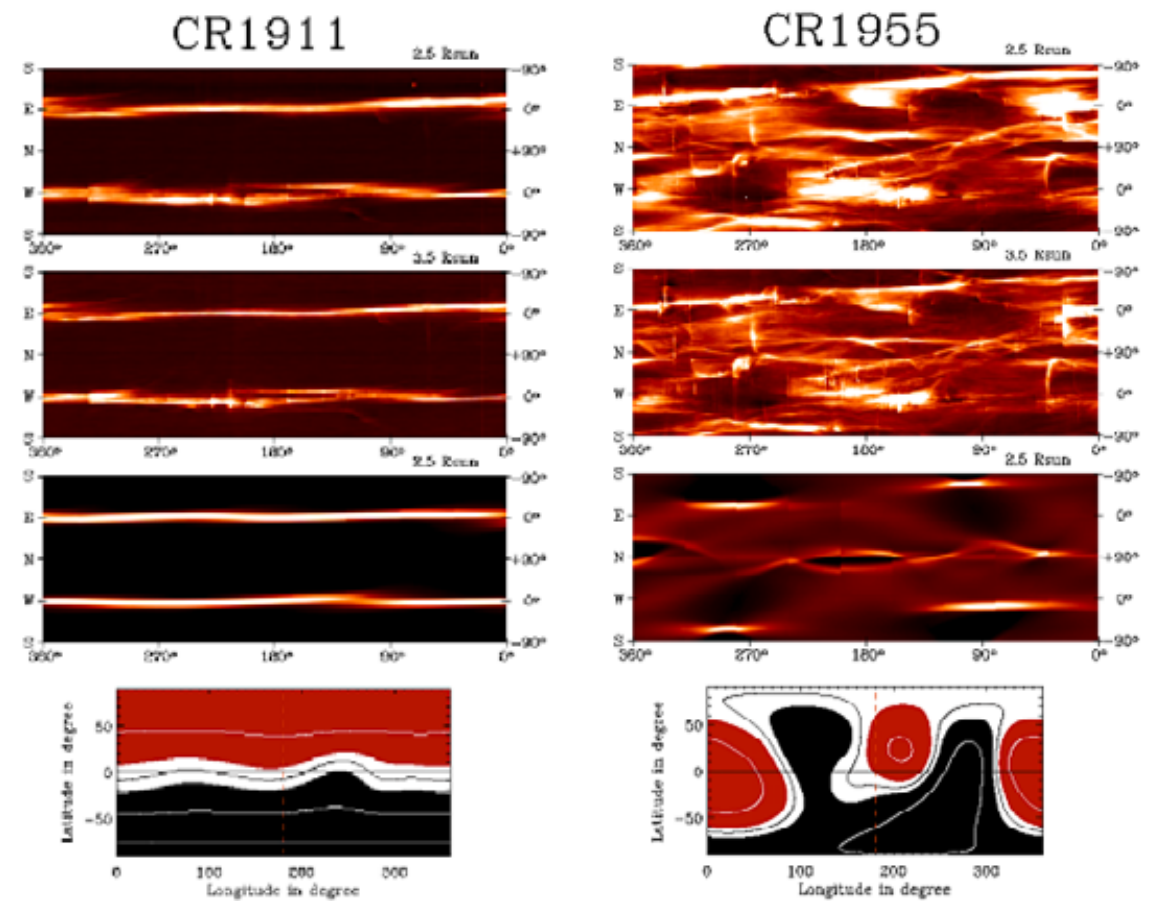

Figure 1. Examples of synoptic maps from observed and simulated images during the CR 1911 on the left and CR 1955 on the right.
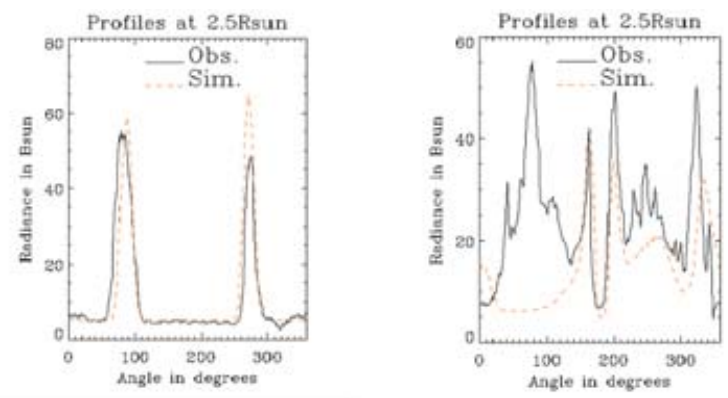

Figure 2. Example of radiance profiles extracted from the observed and simulated synoptic maps covering the period of CR 1910 on the left and CR 1958 on the right.

spacecraft is at increasing latitudes increasing from $0^{\circ}$ to $35^{\circ}$ (nominal and extended missions), and further extrapolated to $90^{\circ}$.

\section{Conclusions}

We have presented a new forward modeling method which allows to simulate the streamer belt as a function of time and solar activity. This method is able to provide both qualitative and quantitative results. The results generally show a good ageement between the observations and the simulation but reveal some unsimulated structures proeminent during the period of the maximum of activity. These structures can be explained by addtional folds or ramifications of the plasma sheet. Our method is flexible and powerfull enough to simulate coronographic images for any solar missions. 


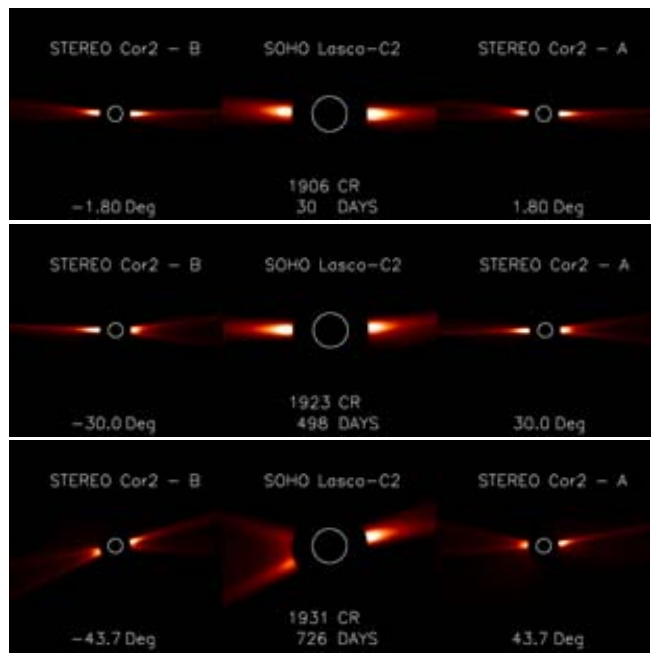

Figure 3. Simulated images corresponding to the view of the STEREO Cor-2 A and B (left and right) and to the view of the $\mathrm{SOHO} \mathrm{LASCO} / \mathrm{C} 2$ in the middle, for three different configurations of the solar corona (CR 1906, CR 1923, and CR 1931) and three angular separations from the Earth $\left(1.8^{\circ}, 30^{\circ}\right.$, and $\left.43.7^{\circ}\right)$.
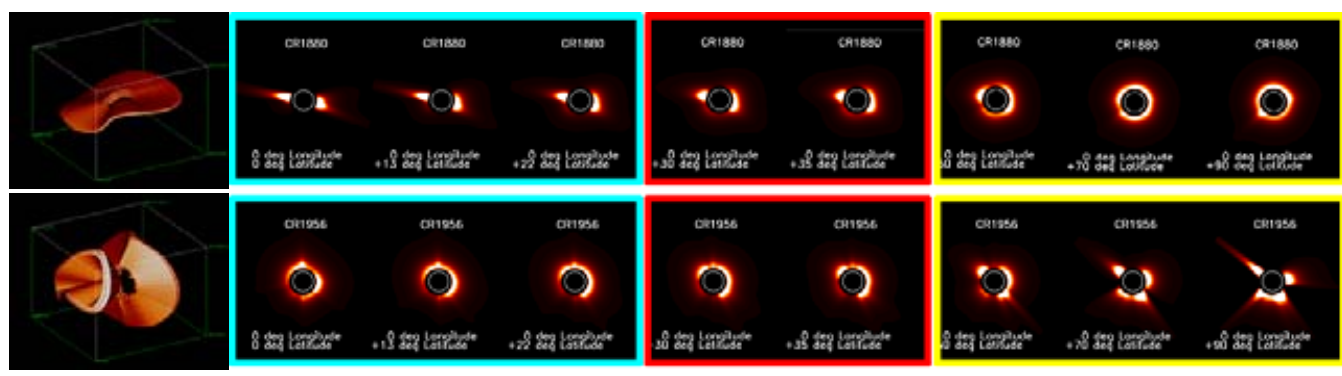

Figure 4. Computed images from static corona using the CR 1880 (top) and CR 1956 (bottom) configurations of the streamer belt, assuming an increasing latitude ranging from $0^{\circ}$ to $90^{\circ}$.

\section{Acknowledgements}

NSO/Kitt Peak data used here are produced cooperatively by NSF/NOAO, NASA/GSFC, and NOAA/SEL.

\section{References}

Bohlin, J. D., \& Garrison, L. M. 1974, Sol. Phys., 38, 165

Koutchmy, S. 1971, Aap, 13, 79

Koutchmy, S., Ershov, A. V., \& Molodenskii, M. M. 1996, Astronomy Reports, 40, 109

Saez, F. , Ph. D Thesis Universit Aix-Marseille I 2005

Saez, F., Zhukov, A. N., Lamy, P., \& Llebaria, A. 2005, Aap, 442, 351

Saito, K., Hata, S., \& Tojo, A. 1972, Annals of the Tokyo Astronomical Observatory, 13, 91

Schatten, K. H., Wilcox, J. M., \& Ness, N. F. 1969, Sol. Phys., 6, 442

Wang, Y.-M., et al. 1997, ApJ, 485, 875

Wang, Y.-M., Sheeley, N. R., \& Rich, N. B. 2000, GRL, 27, 149 